\title{
La habilidad pedagógica de planificar: Una necesidad de los maestros que imparten Educación Física.
}

Raúl Orlando Figueroa Soriano *

\section{RESUMEN}

El presente artículo es el resultado de un estudio experimental (Pre experimento $<$ Pre test y post test>) respecto a la habilidad pedagógica de planificar. El objetivo del estudio es determinar la habilidad de planificar que poseen los maestros de Educación Primaria en lo que respecta al primer ciclo en 6 Centros Básicos de Danlí. De esta manera proponemos un sistema de acciones y operaciones que coadyuven a mejorar la actividad docente. La población es de 120 docentes, cuya muestra fue de 36 enseñantes seleccionados de forma intencional. Son varios los autores que hacen referencia a esa habilidad, misma que todo docente debe dominar para ejecutar el proceso educativo de forma adecuada. Para este estudio consideré fundamentos prestigiosos, y a partir de ellos seleccioné un grupo de acciones y operaciones. Tales acciones y operaciones proponen un accionar con orientaciones metodológicas para maestros de Educación Primaria responsables de impartir la asignatura de Educación Física, correspondiente al Diseño Curricular Nacional Básico de Honduras en su Primer Ciclo. Los resultados del estudio evidenciaron problemática en el desarrollo de esta habilidad. La propuesta fue de gran ayuda para mejorar la actividad docente, pues a posteriori se evidenciaron mejorías a través de los métodos de investigación aplicada, correspondientes a los niveles empíricos, teóricos y matemáticos.

\section{Palabras clave: Habilidad pedagógica de planificar, orientaciones metodológicas, actividad docente.}

\section{ABSTRACT}

The present article is the result of an experimental study (Pre experiment $<$ Pre test and post test>) regarding the pedagogic ability of planning. The objective of the study is to determine the ability to plan that the teachers of Primary Education possess in what concerns to the first cycle in 6 Basic Centers of Danlí. This way we propose a system of actions and operations that cooperate to improve the educational activity.

* Universidad Nacional Autónoma de Honduras - Tecnológica de Danlí. Profesor Titular II.

pitolamaquina2@hotmail.com 
The population is of 120 educational whose sample was of 36 selected educational in an intentional way. They are several the authors that make reference to that ability, same that all educational one should dominate to execute the educational process in an appropriate way. The author of this study assumed approaches of noted foundations, and starting from it selected it a group of actions and operations. That they intend a to work in methodological orientations; for responsible teachers of Primary Education of imparting the subject of Physical Education, corresponding to the Basic National Curricular Design of Honduras in their First Cycle. The results of the study evidenced problem for the development of that ability. The proposal was of great help to improve the educational activity. To posteriori they were evidenced through the methods of applied investigation, corresponding to those of the empiric, theoretical and mathematical level.

Key words: Pedagogic ability to plan, methodological orientations, educational activity. 


\section{INTRODUCCIÓN}

La presente investigación es de naturaleza experimental, comprometida con el perfeccionamiento del Diseño Curricular Nacional Básico (DCNB) en su primer ciclo para el área de Educación Física en la República de Honduras. Actualmente, este diseño presenta virtudes y deficiencias en cuanto a la realidad de la preparación recibida por los maestros que imparten la asignatura de Educación Física en las escuelas primarias de nuestra nación.

Después de una indagación diagnóstica exhaustiva del DCNB se determinó que uno de los componentes de dicho diseño (Las Orientaciones Metodológicas, OM) están elaboradas de forma muy general y no especifican el tratamiento de los contenidos para cada uno de los grados a los que van dirigidas. Lo anterior implica una informatividad metodológica insuficiente para la planificación de la actividad docente, por lo que se concluyó en la necesidad de perfeccionar las OM.

Para esta investigación se trabajó con una población y unas muestras representativas que fueron seleccionadas de forma intencional como se evidencia en el cuadro del capítulo 2: Sobre el tratamiento metodológico de la habilidad pedagógica de planificar. Se seleccionaron y aplicaron métodos del nivel empírico, estadístico y teórico a fin de recopilar los datos necesarios, procesarlos cuantitativamente e interpretarlos en función de la toma de decisiones. La gráfica de ese proceso se muestra en el capítulo 2. Las OM perfeccionadas para los maestros de Educación Primaria se complementan con 12 acciones recomendadas en esta disciplina para la planificación de la actividad docente que generaron valiosos resultados en su aplicación.

Esta indagación hace una contribución a la teoría curricular de la Educación Física; demostrando que los fundamentos teóricos referenciales de esta teoría, considerados en esta investigación, se cumplen también en el plano particular de esta asignatura y en este contexto, por lo que cobran mayor consistencia y validez como teoría general.

\section{DESARROLLO}

\section{Capítulo I. Tratamiento teórico sobre la habilidad pedagógica de planificar.}

La formación y desarrollo de las habilidades pedagógicas profesionales debe ser considerado en la esencia misma del proceso de formación profesional y a ello deben tributar las diferentes actividades que se planifican en el currículo de la 
carrera y fuera de ella, en correspondencia con el seguimiento metodológico. Al abordar las habilidades que debe poseer un docente, la prestigiosa DraC. González. V afirma que todo maestro debe "dominar también muchas otras habilidades que le permitirán llevar a cabo en la forma requerida y con calidad su actividad profesional, por ejemplo: Las habilidades para planificar el contenido del proceso docente educativo, para organizar y dirigir el mismo, para observar y conocer a sus alumnos y establecer una adecuada comunicación con ellos"(1).

La opinión de la DraC. González llama la atención hacia algo importante y es precisamente el enfoque sistémico de la actividad pedagógica profesional. De esta consideración se pueden resumir algunas ideas esenciales que podrán ser utilizadas para una propuesta determinada. Se deben tener en cuenta las características de los sujetos para motivarlos y hacerlos más conscientes de la actividad profesional que están realizando. Se requiere conocer los contextos, métodos, estrategias para su realización; así como la relación con otras habilidades; de ahí su carácter de sistema. Sin embargo, para su tratamiento desde el punto de vista metodológico, pueden considerarse por separado.

Al investigar sobre la habilidad pedagógica de planificar, es de suma importancia considerar que la DraC Hechavarria. M 1996, argumenta que "el profesor debe expresar su actividad pedagógica proyectándola en cinco habilidades pedagógicas fundamentales de la Educación Física y el Entrenamiento Deportivo" (2). En su clasificación se nombran de la siguiente forma: habilidad de proyectar, habilidad constructiva o de planificación, habilidad gnóstica, organizativa y comunicativa. De éstas explicaré las dos primeras por estar ligadas más estrechamente a la temática investigada.

- Habilidad de proyectar: permite visualizar de forma anticipada el sistema interrelacionado de objetivos educativos formativos para el desarrollo de las capacidades físicas y las habilidades motrices que hay que lograr a corto, mediano y largo plazo; durante el proceso docente educativo de la Educción Física.

- Habilidad constructiva o de planificación: se manifiesta en los procedimientos que utiliza el maestro para el ordenamiento de la composición, de los contenidos que debe asimilar el alumno y de las actividades relacionadas con la Educación Física y el Entrenamiento Deportivo. En este caso se debe tener en cuenta el dominio de la habilidad gnóstica para que, a partir de ella, se puedan ejecutar las acciones pedagógicas necesarias y llevar a vías de hecho la planificación de la actividad docente. 
Esta habilidad representa uno de los aspectos más importantes que debe adquirir el maestro que se forma en la Cultura Física ya que tiene que ver con la selección, el orden y la planificación del proceso de la Educación Física Escolar; y la propia planificación de su actividad como maestro. Rosales, A. 2002 argumenta que "cuando se habla de planificación se asocia a la idea de organizar, ordenar, coordinar, prever, etc. Una de la ideas centrales que aparecen cuando hablamos de planificación es la de fijar cursos de acción con el propósito de alcanzar determinados objetivos mediante el uso eficiente de estrategias y medios para su concreción" (3).

La misma autora plantea: "Planificar es la acción consistente en utilizar un conjunto de procedimientos mediante los cuales se introduce una mayor racionalidad y organización en un conjunto de actividades y acciones articuladas entre sí, previstas anticipadamente. Tiene el propósito de influir en el curso de determinados acontecimientos, con el fin de alcanzar una situación elegida como deseable, mediante el uso eficiente de medios y recursos escasos o limitados" (4).

Las funciones que cumple esta habilidad para el docente pueden sintetizarse en los siguientes aspectos: anticipar, prever, organizar, aclarar, secuenciar, adecuar el grupo y la institución. También permite modificar, si es necesario, lo seleccionado; intentando encontrar un equilibrio entre la sobrevalorización y la desvalorización de la planificación didáctica.

En las bibliografías consultadas, donde se trata la actividad docente como forma fundamental de organizar el proceso docente educativo, se refiere a la importancia de la clase de Educación Física Escolar por la unidad que se logra en ella de lo físico, lo instructivo y lo educativo; atendiendo a lo relacionado con la ejecución de la misma pero no a cómo se planifica.

Sin embargo, en el estudio realizado por la DraC Hechavarría, M. 2006 sobre las habilidades pedagógicas profesionales "relaciona un conjunto de habilidades que deben formarse y desarrollarse en el docente, las cuales guardan relación con las declaradas por la Doctora González Maura" (5), entre ellas:

- Habilidad necesaria para planificar el trabajo docente educativo (Actividad docente).

- Habilidad para planificar su propia actividad.

- Habilidad para planificar el proceso de entrenamiento deportivo.

- Habilidad para transmitir la información.

- Habilidad para planificar la clase (Actividad docente). 
Esta indagación se centró en el estudio de la primera y la última, ambas de interés tanto para el maestro formado, como para el que está en formación; así como lo indica la DraC. Hechavarría, M. 2006 quien argumenta "que si bien las habilidades siempre se inician a partir de los conocimientos y se apoyan en ellos, constituye ante todo, el estado de disposición para el desempeño de funciones teóricas y prácticas que relacionamos a continuación" (6).

Después de sistematizar el criterio de varios autores, se propone un conjunto de acciones inmersas en un conjunto de orientaciones metodológicas para mejorar la habilidad pedagógica de planificar. Tomando en cuenta la realidad de la actividad docente que se desempeña en Honduras, esta habilidad compromete las siguientes acciones reconocidas en el perfil profesional:

1. Utilización de la información contenida en los documentos que determinan y orientan el desarrollo del proceso de Educación Física; incluyendo los que orientan el desarrollo de la preparación metodológica de la cátedra.

2. Aplicación de los principios fundamentales de la planificación.

3. Interpretación de los estándares y expectativas de logro de la Educación Física en los diferentes grados.

4. Interpretación de los estándares y las expectativas de logro específicas de cada bloque de contenidos y destacar los aspectos fundamentales.

5. Aplicación de la concepción metodológica de cada bloque de contenidos que aparece en las OM.

6. Determinar y disponer de las condiciones materiales y los medios auxiliares que son necesarios para el desarrollo efectivo de cada bloque.

7. Análisis metodológico de los contenidos programados; derivando la estructura de cada movimiento en sus acciones y operaciones componentes; teniendo en cuenta los estándares, las expectativas de logro propuestas en la asignatura, la habilidad principal, las habilidades antecedentes, las capacidades físicas que faciliten el desarrollo, los conocimientos teóricos y la sistematización de los ejercicios.

8. Distribución del contenido global o parcial del curso o bimestre (Plan temático); teniendo en consideración los aspectos siguientes:

- Cantidad de frecuencias y horas lectivas, así como su distribución entre los diferentes bloques de contenidos, sub. bloques de contenidos y así como las capacidades a desarrollar en cada uno de ellos.

- La integración de los contenidos.

9. Elaboración de las fichas pertenecientes a los test/diagnósticos que aplicarán al inicio del curso, al final del segundo y quinto bimestre (este paso 
corresponde con las OM del bloque condición física para la salud, habilidades y destrezas básicas).

10. Confección del sistema de clases para cada bimestre sobre la base del diagnóstico aplicado y el análisis metodológico realizado. El sistema de clases contempla estándares, expectativas de logro, actividades, métodos, procedimientos organizativos, medios y evaluación.

11. Planificación de cada clase e introducir los ajustes de contenidos y/o métodos obtenidos de valoración de los resultados de cada clase en correspondencia con la forma en que los alumnos logran los estándares y expectativas. Para ello deberá tener en cuenta principalmente los siguientes aspectos:

- Consideración de los resultados del diagnóstico para el pronóstico.

- Utilización de la documentación que determina el desarrollo de la Educación Física.

- Utilización de la bibliografía en correspondencia con el contenido a desarrollar.

- Selección adecuada de los ejercicios de calentamiento general y específico.

- Selección adecuada de los ejercicios preparatorios.

- Interrelación de los aspectos instructivos y educativos en la clase.

- Integración intra e interdisciplinaria de los contenidos con otras áreas del currículo.

- Establecimiento del estilo de enseñanza predominante en la clase.

- Aplicación del principio del aumento gradual y progresivo de la carga a lo largo de las clases.

- Aplicación de los principios didácticos y pedagógicos generales.

- Selección apropiada de tareas extra clase.

- Selección de actividades de evaluación de conocimientos.

- Selección de formas de control del rendimiento físico y cognitivo de los alumnos.

- Precisión de cómo valorar el resultado de su trabajo al concluir la clase.

- Determinar las tareas de trabajo independiente, en correspondencia con las funciones didácticas pretendidas.

12. Comprobación, mediante controles sistemáticos, del logro de las expectativas programadas y tomar medidas necesarias que se deriven del análisis de sus resultados, y así garantizar una dirección adecuada del proceso educativo docente. 
Las acciones anteriores, devenidas en habilidades, se tomaron en cuenta en las valoraciones de las OM existentes y en las que se propusieron para mejorar la informatividad metodológica de la planificación de la actividad docente y el desarrollo del proceso docente educativo de la asignatura en el "Primer Ciclo".

En esta indagación se asume como definición de Habilidad Pedagógica Profesional de Planificar el constructo de Domínguez, M. 2007:

"Las acciones y operaciones que realiza el maestro dirigidas a diseñar de forma anticipada el proceso docente - educativo, basado en el programa escolar y orientaciones metodológicas donde se concretan los procesos estructurales, se definen los objetivos y se distribuyen las tareas y actividades a realizar en la clase para alcanzar dichos objetivos de acuerdo a las condiciones y el nivel de desarrollo de los estudiantes"(7).

Esta definición tiene implicaciones importantes para la estructuración de la habilidad pedagógica de planificación de la actividad docente en Educación Física. Por tanto, los componentes estructurales tienen en cuenta factores importantes como los objetivos, la distribución de los contenidos, las acciones y las condiciones. Algunos aspectos son conocidos al interior de la definición, pero aún así se subraya que el objetivo es el modelo pedagógico del encargo social, la aspiración, el propósito que la institución docente se propone alcanzar en los alumnos y por ende orienta la educación de los ciudadanos que en ella se forman.

Los contenidos comprenden la parte de la cultura humana propia de la disciplina comprometida con los aprendizajes que los alumnos deben alcanzar para progresar en las direcciones que marcan los fines de la educación en una etapa de la escolarización, en cualquier área o fuera de ella, para lo que es preciso estimular comportamientos, adquirir valores, actitudes, habilidades y conocimientos. La distribución de tareas se refiere al contenido específico que se trabaja en la clase. El mismo debe estar dosificado de forma gradual de acuerdo a su complejidad y al nivel de desarrollo de los alumnos. En el caso de la Educación Física Escolar es necesaria, además, la distribución de ejercicios, juegos y competencias; teniendo en cuenta los principales componentes de la carga física a considerar para los mismos.

Como las habilidades pedagógicas profesionales se expresan de forma fenomenológica en un saber hacer, y para ello se necesitan los conocimientos asociados a éstas, entonces podemos llamarles conocimientos previos, tanto a los generales como a los específicos. Éstos son aportados, como se explicó 
anteriormente, por las diferentes asignaturas del currículo. En el caso de los conocimientos generales, se consideran aquellos relacionados con las principales ciencias afines como Morfología, Fisiología, Biomecánica, entre otras; y los conocimientos específicos, aportados por las asignaturas del ejercicio de la profesión, tales como Pedagogía, Teoría y Metodología de la Educación Física; que tributan directamente la habilidad pedagógica de planificación de la actividad docente.

\section{Capítulo II. Tratamiento metodológico de la habilidad pedagógica de planificar.}

Para determinar y cumplir el objetivo de estudio se aplicaron métodos y técnicas de investigación, así como se puede observar en el esquema que se establece y es explicado a continuación, referido a todo el proceso investigativo.

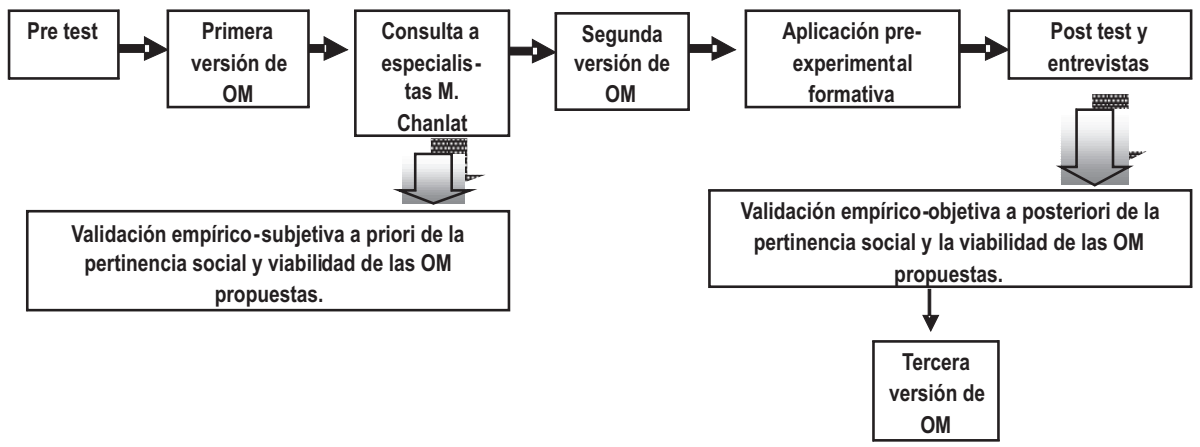

- En primera instancia se aplicó un pre test (Cuestionario) donde se demostró la problemática acerca de la habilidad pedagógica de planificar.

- Luego se hizo una primera versión del accionar para mejorar la habilidad pedagógica de planificar. Posteriormente se sometió ese accionar a una consulta de especialistas en Educación Física (Matriz de Chanlat).

Después de hacer la consulta a especialistas, se hizo una segunda versión del accionar para mejorar la habilidad pedagógica de planificar. Seguido a ello, esas acciones se aplicaron a través de un pre-experimento formativo. Inmediato a lo anterior se aplicó un cuestionario a los docentes para determinar el resultado de las acciones elaboradas para determinar la habilidad anteriormente dicha; tomándose en cuenta la opinión de ellos para fijar la versión propuesta.

Todo el proceso investigativo se llevó a cabo con la siguiente población y muestra 


\begin{tabular}{|c|c|c|c|c|}
\hline $\begin{array}{c}\text { Población y Muestra: } \\
\text { Estratos }\end{array}$ & Población & Muestra & $\%$ & $\begin{array}{c}\text { Tipo de } \\
\text { muestreo }\end{array}$ \\
\hline Maestros & 120 & 36 & 30 & Intencional \\
\hline Directivos & 12 & 6 & 50 & Intencional \\
\hline Especialistas (S.E.) & 6 & 6 & 100 & Intencional \\
\hline Especialistas (E.F) & 20 & 20 & 100 & Intencional \\
\hline
\end{tabular}

A continuación se presentan los resultados de correlación del pre test y post test.

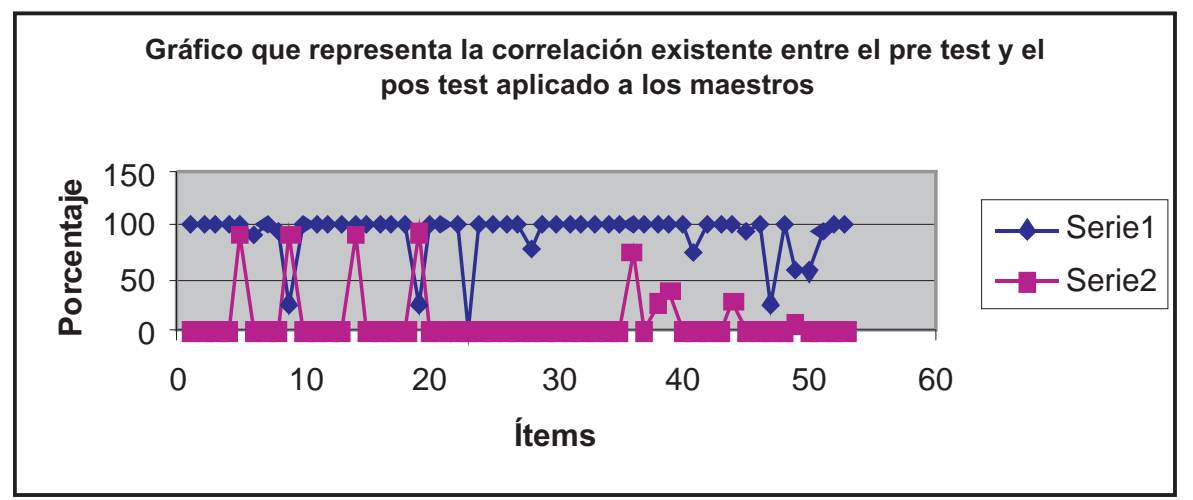

En correspondencia a la comparación establecida entre el instrumento \# 1 y el instrumento \# 3, conforme a los ítems del 1-53 de cada instrumento, se pudo constatar un coeficiente de correlación de - 0.2853; indicando de esta manera la correlación inversamente proporcional del signo esperado entre las comparaciones hechas. Todo lo anterior conforme a la habilidad pedagógica de planificar y respecto a lo establecido en la etapa diagnóstica y la etapa de post cuasi experimento. Dicho aspecto indica la mejoría de la habilidad de planificar después de la intervención.

Mediante la aplicación de este instrumento (Encuesta) se constató el mejoramiento de la informatividad metodológica de planificar la actividad docente, y a su vez, las acciones y operaciones de la habilidad pedagógica de planificar; pues al ejecutar un análisis cualitativo y cuantitativo en más de un $90 \%$, los maestros de Educación Primaria, encargados de dirigir el Primer Ciclo y que fueron muestra de esta investigación, evidenciaron el cambio de esta habilidad durante el proceso de investigación. 


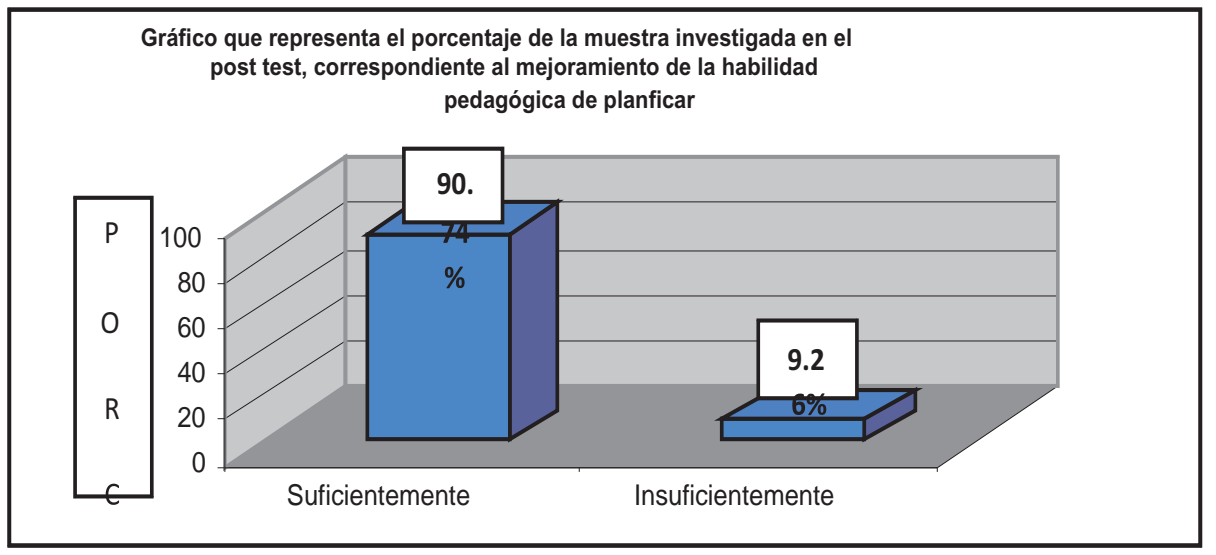

\section{CONCLUSIONES}

1. Los resultados de la sistematización realizada por varios autores especialistas en temas sobre Cultura Física y en la estructuración de orientaciones metodológicas; así como en la temática sobre la habilidad pedagógica de planificar, permitieron determinar que la habilidad de planificar se forma y se desarrolla en la actividad profesional de los sujetos. Es por ello que para entender la magnitud de su complejidad y los modos de su desarrollo debe comprenderse la categoría de actividad como lo más general.

2. El accionar elaborado mediante la sistematización de varios autores y que su vez fue perfeccionado después de haber sido verificada la problemática sobre la habilidad pedagógica de planificar mediante un cuestionario perteneciente al proceso experimental de un pre test y post test; y además retroalimentado a través de una encuesta aplicada a los maestros del primer ciclo de primaria, encargados de dirigir el proceso docente educativo de la asignatura Educación Física (muestra), evidenció el cambio sustancial de la problemática que existía en cuanto a esa habilidad.

\section{BIBLIOGRAFÍA}

Domínguez, María Elena. (2007). Propuesta para la preparación metodológica del maestro en formación orientada al desarrollo de la habilidad pedagógica profesional de planificar la clase de Educación Física escolar. Tesis post grado, Maestría en Educación Física, Instituto Superior de Cultura Física Manuel Fajardo, La Habana. p. 56. 
González Maura, V. (1995). Psicología para educadores. 1ra. ed. La Habana: Editorial Educación. p. 117.

Hechavarria Urdaneta, M. (1996). Criterio didáctico metodológico sobre las habilidades pedagógicas profesionales a la luz de la tendencia contemporánea de la Educación Física. Documento impreso. Instituto Superior de Cultura Física Manuel Fajardo "ISCFMF".La Habana. p. 9.

Hechavarría Urdaneta, Mirtha. (2006). Lo que todo entrenador deportivo y director técnico debe saber hacer. Documento impreso. Instituto Superior de Cultura Física Manuel Fajardo "ISCFMF”.La Habana. p. 74.

Hechavarría Urdaneta, Mirtha. (2006). Lo que todo entrenador deportivo y director técnico debe saber hacer. Documento impreso. Instituto Superior de Cultura Física Manuel Fajardo "ISCFMF".La Habana. p. 74.

Rosales, Analia. (2002). La planificación de aula, patio en educación física. Documento impreso. Instituto Superior de Cultura Física Manuel Fajardo "ISCFMF".La Habana. p. 1.

Rosales, Analia. (2002). La planificación de aula, patio en educación física. Documento impreso. Instituto Superior de Cultura Física Manuel Fajardo "ISCFMF".La Habana. p. 1. 